\title{
METODOLOGIAS DE COLETA NA AVALIAÇÃO DA COMUNIDADE EPÍGEA EM FRAGMENTO FLORESTA CILIAR
}

\author{
Joseane Aparecida Derengoski ${ }^{1}$, Barbara Elis Santos Ruthes²*, Daiane Cristina Zanellato ${ }^{3}$, Dinéia \\ Tessaro 4 \\ ${ }^{1}$ Doutoranda em Agronomia, Universidade Tecnológica Federal do Paraná - Câmpus Pato Branco (UTFPR-PB); ${ }^{2}$ Doutoranda \\ em Agronomia, Universidade Federal do Paraná (UFPR); ${ }^{3}$ Mestre em Agroecossistemas; ${ }^{4} \mathrm{Dr}^{\mathrm{a}}$ em Engenharia Agrícola, \\ professor Adjunto Universidade Tecnológica Federal do Paraná - Câmpus Dois Vizinhos (UTFPR-DV);
}

RESUMO: Os ecossistemas florestais exercem papel fundamental em inúmeros processos que envolvem a manutenção e a qualidade de vida dos organismos edáficos. Contudo, isolar, identificar e quantificar estes indivíduos ainda é considerado uma tarefa difícil, sendo empregadas diversas técnicas que auxiliam na sua captura. Assim, o presente trabalho teve como objetivo identificar a comunidade epígea em um fragmento florestal utilizando os métodos Pitfall e Provid para coleta da fauna epígea. Foram instaladas pela floresta, 12 armadilhas de cada metodologia, contendo solução conservante de formol a 4\%, permanecendo a campo por sete dias. Os organismos foram triados e classificados ao nível de ordem com auxílio de estereomicroscópio. As abundâncias e riquezas obtidas foram submetidas a testes de normalidade e homogeneidade de variância e as metodologias comparadas entre si por teste de Tukey a $5 \%$ de probabilidade e análise de componentes principais. Foram calculadas a frequência relativa e os índices ecológicos de Shannon-Wiener, equabilidade de Pielou, riqueza de Margalef e dominância de Simpson. Não houve diferenças significativas entre as metodologias avaliadas para coleta da comunidade epígea, sendo possível utilizar o método Pitfall com a mesma eficiência do método Provid.

Palavras-chave: fauna do solo, pitfall, provid

\section{IDENTIFICATION OF THE EPIGEAL COMMUNITY IN FOREST FRAGMENT BY DIFFERENT COLLECTION METHODS}

ABSTRACT: Forest ecosystems play a fundamental role in numerous processes that involve the maintenance and quality of life of soil organisms. However, isolating, identifying and quantifying these individuals is still considered a difficult task, using several techniques that help in their capture. Thus, the present work had as objective to identify the edaphic community in a forest fragment using the methods Pitfall and 
Provid to collect the fauna epigeal. Twelve traps of each methodology, containing $4 \%$ formol preservative solution, were installed in the forest, remaining in the field for seven days. The organisms were sorted and classified at the order level with the aid of a stereomicroscope. The abundances and richness obtained were subjected to normality tests and homogeneity of variance and the methodologies compared to each other by Tukey test at 5\% of probability and principal components analysis. The relative frequency and ecological indexes of Shannon-Wiener, Pielou equability, Margalef richness, and Simpson dominance were calculated. There were no significant differences between the methodologies evaluated for the collection of the epigene community, being possible to use the Pitfall method with the same efficiency of the Provid method.

Keywords: soil fauna, pitfall, provid

\section{INTRODUÇÃO}

A biodiversidade de um ecossistema tem correlação íntima e positiva com a sustentabilidade e com o equilíbrio do ambiente, sendo uma das propriedades fundamentais da natureza responsável por manter a estabilidade dos ecossistemas (BARRETA et al., 2011). A investigação desta biodiversidade avançou nos últimos anos e a consciência entre cientistas e a comunidade política vêm crescendo sobre a importância da biodiversidade do solo na prestação de serviços ecossistêmicos à sociedade humana (WALL et al., 2012; BARDGETT; PUTTEN, 2014).

Em florestas sob diferentes níveis de conservação, o estudo da comunidade da fauna edáfica é o início do entendimento dos processos que ocorrem no solo, visto que estes organismos ocupam diversos níveis tróficos na cadeia alimentar. Dentre as funções essenciais que desempenham estão a decomposição da matéria orgânica, ciclagem de nutrientes, melhoria nas condições físicas do solo, aumento da porosidade e por consequência, infiltração de água, além de exercer papel fundamental na estrutura do solo e agregação (BARETTA et al., 2010; BARETTA et al., 2011; HUERTA; WAL, 2012; BARDGETT; PUTTEN, 2014).

Para o estudo destes organismos devido ao seu pequeno tamanho, 0 procedimento consiste na captura, identificação e quantificação dos indivíduos que ocorrem na comunidade (FREIRE et al., 2015), sendo utilizadas diversas metodologias de forma a capturar a maior biodiversidade possível. Uma das armadilhas de solo mais utilizadas é a do tipo Pitfall trap's (armadilha de queda), 
destinada à captura de organismos que se locomevem sobre o solo (fauna epígea) (ARAÚJO et al., 2010), tais como Collembola, Protura, Diplura, Formicidae, Acari, Araneae, Diplopoda, entre outros.

As armadilhas do tipo Provid surgiram como opção prática, econômica e de rápida instalação, além de possuir eficiência semelhante aos métodos comumente utilizados. São constituídas por recipientes de plástico, com aberturas na forma de janelas nas laterais, enterradas ao solo de modo que suas bordas fiquem niveladas a superfície. Da mesma forma com o que ocorre na armadilha Pitfall, a coleta dos organismos ocorre por meio de interceptação e queda (ANTONIOLLI et al, 2006).

Em termos metodológicos, não há um único método capaz de amostrar, eficientemente e, ao mesmo tempo, a meso e a macrofauna edáfica (BARETTA et al., 2011). Na prática, as diferentes metodologias para cada classe de tamanho significam que um estudo raramente vai contemplar a avaliação da meso e macrofauna simultaneamente (CORREIA; ANDRADE, 2008). Neste sentido, devido à escassez de trabalhos que comparem as duas armadilhas, o presente trabalho objetivou classificar a comunidade epígea de um fragmento florestal por meio de diferentes métodos de coleta.

\section{MATERIAL E MÉTODOS}

A área de estudo localiza-se na estação experimental da Universidade Tecnológica Federal do Paraná, Câmpus Dois Vizinhos - PR, entre as coordenadas geográficas $25^{\circ} 41^{\prime} 30^{\prime \prime S}$ e $53^{\circ} 06^{\prime} 04^{\prime \prime} \mathrm{O}$, sendo uma floresta ciliar com área total de 48 hectares. O clima local é classificado como subtropical úmido mesotérmico (Cfa), sendo a temperatura média, no mês mais frio, inferior a $18{ }^{\circ} \mathrm{C}$ e no mês mais quente, acima de $22{ }^{\circ} \mathrm{C}$, com verões quentes. A umidade relativa varia entre 64 a $74 \%$ e as chuvas são bem distribuídas durante todo 0 ano, com precipitação pluviométrica entre 1800 a $2200 \mathrm{~mm}$ anuais (ALVARES et al., 2013).

Apresenta altitude média entre 475 e 510m com prredominância de Latossolo Vermelho (EMBRAPA, 2013). A vegetação original é classificada como uma área de transição entre a Floresta Ombrófila Mista e a Floresta Estacional Semidecidual, em 
estágio médio da sucessão florestal, com manchas em estágios inicial e final, sendo que tal floresta já sofreu extração de madeira e plantio de espécies exóticas.

Para amostragem da fauna epígea, foram comparadas duas metodologias, amplamente utilizadas em estudos da fauna do solo. As armadilhas Pitfall utilizadas consistem em recipientes plásticos transparentes, com volume de $750 \mathrm{~mL}$, contendo $300 \mathrm{~mL}$ de solução conservante de formol a $4 \%$ e tensoativo ( $1 \mathrm{~mL} / \mathrm{L})$, que tem o objetivo de quebrar a tensão superficial da água e impedir que os animais retornem à superfície. Já a armadilha Provid (ANTONIOLLI et al., 2006) adaptada, constituiuse de uma garrafa plástica tipo pet, com capacidade de $500 \mathrm{~mL}$, com quatro aberturas na forma de janelas com dimensões de $6 \times 4 \mathrm{~cm}$. Em cada armadilha adicionou-se $150 \mathrm{~mL}$ de solução conservante de formol a $4 \%$ e tensoativo (1 mL/L).

A instalação das armadilhas a campo foi realizada com auxílio de trado para a abertura de buracos no solo, e na sequência, as armadilhas foram enterradas com a borda vazada nivelada ao solo e camuflada com o material orgânico local, de forma que os animais ao se locomoverem, caíssem acidentalmente nesses recipientes. Sobre as armadilhas Pitfall, foi instalada uma estrutura composta de palitos de madeira acoplados a um recipiente de alumínio, para evitar o transbordamento ou diluição da solução com possíveis precipitações (Figura 1).
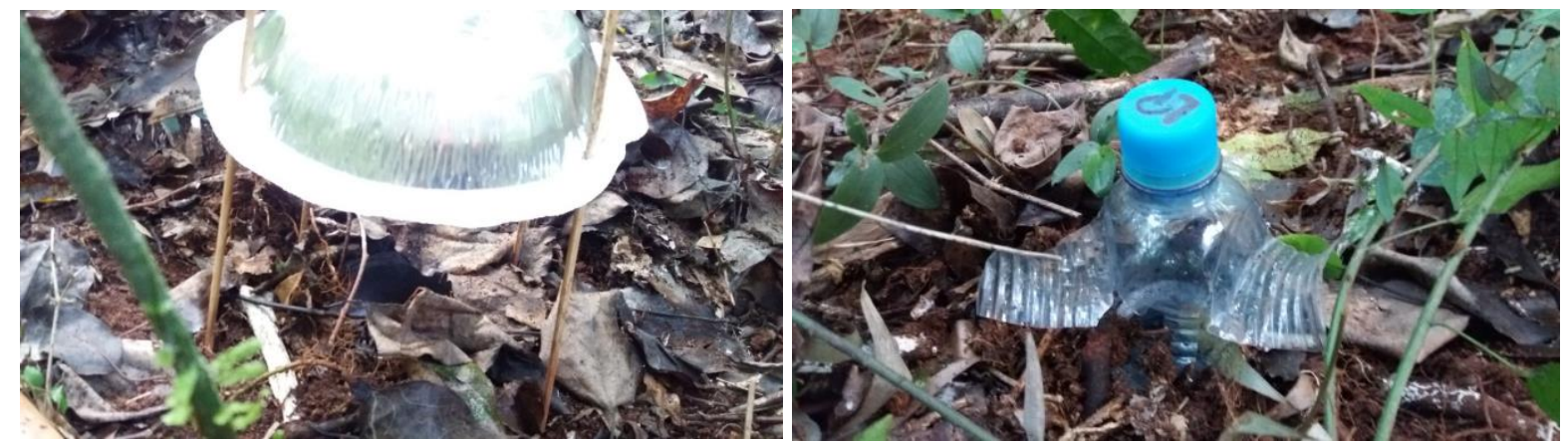

Figura 1 - Armadilhas tipo Pitfall e Provid para amostragem da fauna epigea instaladas na floresta ciliar do Câmpus da UTFPR, em Dois Vizinhos - PR.

Figure 1 - Pitfall and Provid type traps for epigeal fauna sampling in the riparian forest of the UTFPR Campus, in Dois Vizinhos - PR.

A amostragem foi realizada em 10 de maio de 2017, instalando-se 12 armadilhas por tratamento, distribuídas pela floresta, espaçadas em 30 metros de 
forma a capturar o máximo de diversidade possível em toda a sua extensão. Em cada ponto amostral, instalou-se uma armadilha Pitfall e uma Provid, distanciadas em aproximadamente $30 \mathrm{~cm}$ entre elas. As armadilhas permaneceram 7 dias a campo, sendo posteriormente recolhidas e levadas ao laboratório, onde foram triadas, armazenadas em frascos contendo etanol a 70\% e classificadas com auxílio do estereomicroscópio, em aumento de 40 vezes ao nível de taxonômico de ordem.

A partir da triagem da fauna epígea, foram obtidos os dados de abundância total de cada grupo taxonômico, riqueza (S) total de grupos taxonômicos e frequência relativa (Equação 1).

$$
r \%=\text { frequência absoluta/total de frequências absolutas }
$$

(Equação 1)

A abundância e riqueza foram submetidas aos testes de normalidade de Lilliefors e Kolmogorov-Smirnov e aos testes de homogeneidade de variância de Hartley, Cochran e Bartlett. Os dados que não atenderam aos pressupostos matemáticos foram transformados em log $x$ e posteriormente submetidos à análise de variância (ANOVA). As duas metodologias de coleta foram comparadas entre si por meio do teste de Tukey ( $p \geq 0.05$ ), utilizando o software R Bio (BHERING, 2017).

A partir dos dados obtidos foram calculados os índices ecológicos de Simpson, pouco influenciado por espécies raras; Shannon-Wiener, que atribui maior peso a espécies raras; Margalef, que estima a biodiversidade da comunidade e Pielou, que indica se a abundância das espécies é iguail ou diferente (MAGURRAN, 2011), os quais foram calculados pelo software Past (HAMMER, 1999-2012).

O índice de dominância de Simpson (D) mede a equitabilidade da comunidade, variando de 0 a 1 , sendo que quanto mais próximo de 0 menor a dominância. É definido pela equação 2 , onde ni é o número de indivíduos do táxon i.

$$
D=1-\sum_{i}\left(\frac{n i}{n}\right)^{2}
$$

O índice de diversidade de Shannon-Wiener $(H)$ varia de 0 a 5, sendo a maior diversidade indicada por valores mais altos, enquanto valores menores indicam 
predominância de uma ou mais espécies no ambiente, diminuindo a diversidade da comunidade (SHANNON, 1948). É definido pela equação 3 , onde $\mathrm{pi}=\mathrm{ni} / \mathrm{N}$; ni = valor de importância de cada espécie ou grupo e $\mathrm{N}=$ total dos valores de importância.

$$
H=-\sum p i * L O G p i
$$

(Equação 3)

O índice de riqueza de Margalef é uma medida utilizada em ecologia para estimar a biodiversidade de uma comunidade com base na distribuição numérica dos indivíduos das diferentes espécies em função do número total de indivíduos existentes na amostra analisada (MAGURRAN, 2011). É definido pela equação 4, onde onde $\mathrm{S}$ é a diversidade, $\mathrm{n}$ é o número de espécies presente, e $\mathrm{N}$ é o número total de indivíduos encontrados (pertencentes a todas as espécies).

$$
M=\frac{S-1}{\ln n}
$$

O índice de equitabilidade de Pielou (e) varia de 0 a 1 , sendo que valores tendendo a 1 indicam maior uniformidade da comunidade, ou seja, todas as espécies são igualmente abundantes (PIELOU, 1966). É definido pela equação 5, onde onde: $\mathrm{H}$ = índice de Shannon e $\mathrm{S}$ = Número de espécies ou grupos.

$$
e=H /(\log S)
$$

(Equação 5)

Com o objetivo de verificar as relações entre os grupos taxonômicos e os tipos de armadilhas utilizadas, realizou-se a análise de componentes principais (ACP) utilizando o programa estatistico PCord 4.0 (MCCUNE; MEFFORD, 1999).

\section{RESULTADOS}

Durante o período de amostragem, a precipitação acumulada no município de Dois Vizinhos foi de 27,6 mm e após esse período de chuva, a temperatura mínima chegou a $10^{\circ} \mathrm{C}$ (Figura 2), o que pode ter contribuído para a redução da atividade da fauna epígea e consequente na captura pelas armadilhas. 


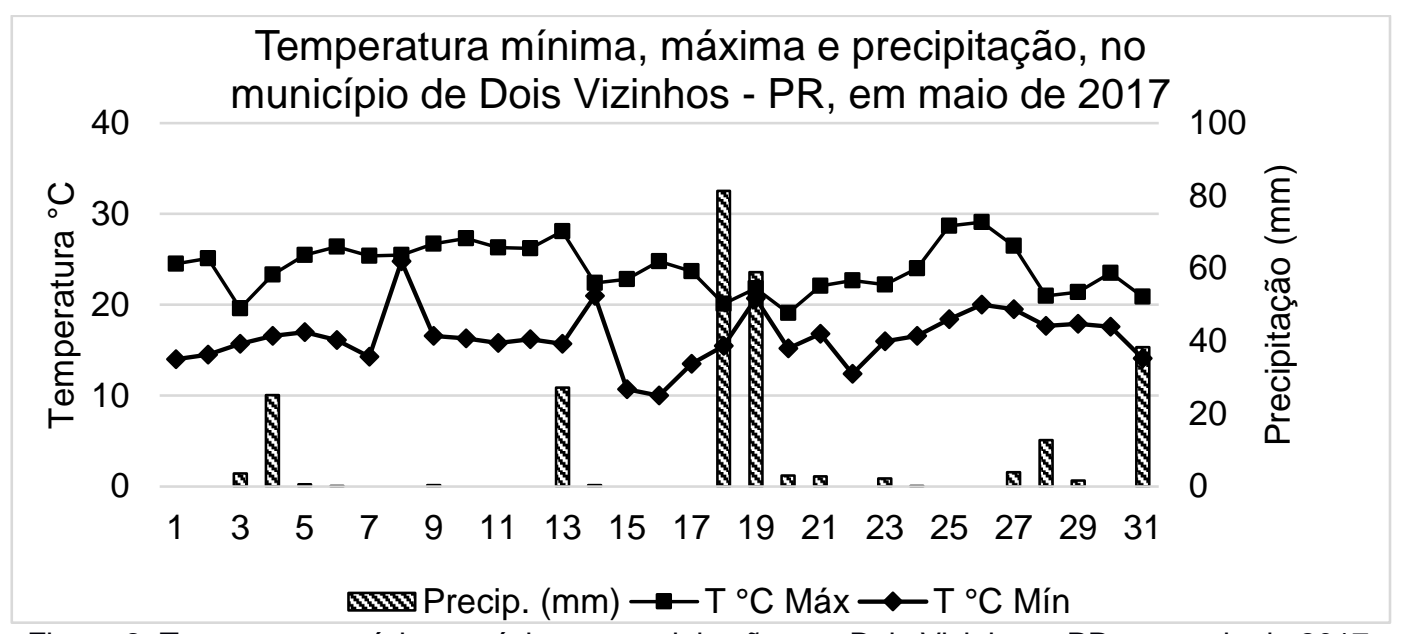

Figura 2. Temperatura mínima, máxima e precipitação, em Dois Vizinhos - PR, em maio de 2017. Figure2. Minimum temperature, maximum and precipitation, in Dois Vizinhos - PR, in May 2017.

Foram amostrados 2.569 indivíduos da fauna epígea, sendo 1.388 coletados com a armadilha Pitfall e 1.181 com a armadilha Provid (Tabela 1).

Tabela 1. Grupos taxonômicos da fauna epígea, abundância, riqueza e diversidade de indivíduos amostrados pelas metodologias de Pitfall e Provid, instaladas na floresta ciliar do Câmpus da UTFPR, em Dois Vizinhos - PR. Table 1. Taxonomic groups of the epigeal fauna, abundance, richness and diversity of individuals sampled by the Pitfall and Provid methodologies, installed in the riparian forest of the UTFPR Campus, in Dois Vizinhos - PR.

\begin{tabular}{cccc}
\hline CLASSE & ORDEM TAXONÔMICA & \multicolumn{2}{c}{ TRATAMENTOS } \\
\cline { 3 - 4 } TAXONÔMICA & Pcariformes & $3^{\text {ns }}$ & $2^{\text {ns }}$ \\
Arachnida & Araneae & $28^{\text {ns }}$ & $23^{\text {ns }}$ \\
Collembola & Collembola & $813^{\text {ns }}$ & $794^{\text {ns }}$ \\
& Blattodea & $8^{\text {ns }}$ & $6^{\text {ns }}$ \\
& Coleoptera & $78^{\text {ns }}$ & $66^{\text {ns }}$ \\
Insecta & Diptera & $299^{\text {ns }}$ & $161^{\text {ns }}$ \\
& Hemiptera & $20^{\text {ns }}$ & $5^{\text {ns }}$ \\
& Hymenoptera & $111^{\text {ns }}$ & $100^{\text {ns }}$ \\
& Lepidoptera & $5^{\text {ns }}$ & $19^{\text {ns }}$ \\
& Orthoptera & $23^{\text {ns }}$ & $1181^{\text {ns }}$ \\
& Abundância & $1388^{\text {ns }}$ & $10^{\text {ns }}$ \\
& Riqueza & $10^{\text {ns }}$ & 0,48 \\
& Simpson & 0,40 & 1,14 \\
\hline
\end{tabular}

${ }^{\text {ns }}$ não significativo ao nível de $5 \%$ de probabilidade de erro.

Os organismos pertencian a 10 grupos taxonômicos diferentes, sendo a maioria $(62,55 \%)$ pertencente à Classe Collembola, seguidos pela Classe Insecta 
$(35,27 \%)$ e pela Classe Arachnida (2,18\%). Não houve diferenças significativas entre os grupos taxonômicos encontrados, abundância ou riqueza de organismos.

Em relação aos índices ecológicos avaliados, a armadilha Pitfall apresentou maior diversidade de organismos $(1,28)$, melhor uniformidade da comunidade $(0,56)$, e menor dominância $(0,40)$ se comparados à armadilha Provid. Em ambos os casos, o índice de Margalef, apresentou valor inferior a 2. As ordens Collembola, Diptera e Hymenoptera foram as mais frequentes em ambas as metodologias estudadas, representando $88,1 \%$ do total coletado pelo método Pitfall e $89,3 \%$ do total coletado pelo método Provid (Figura 3).

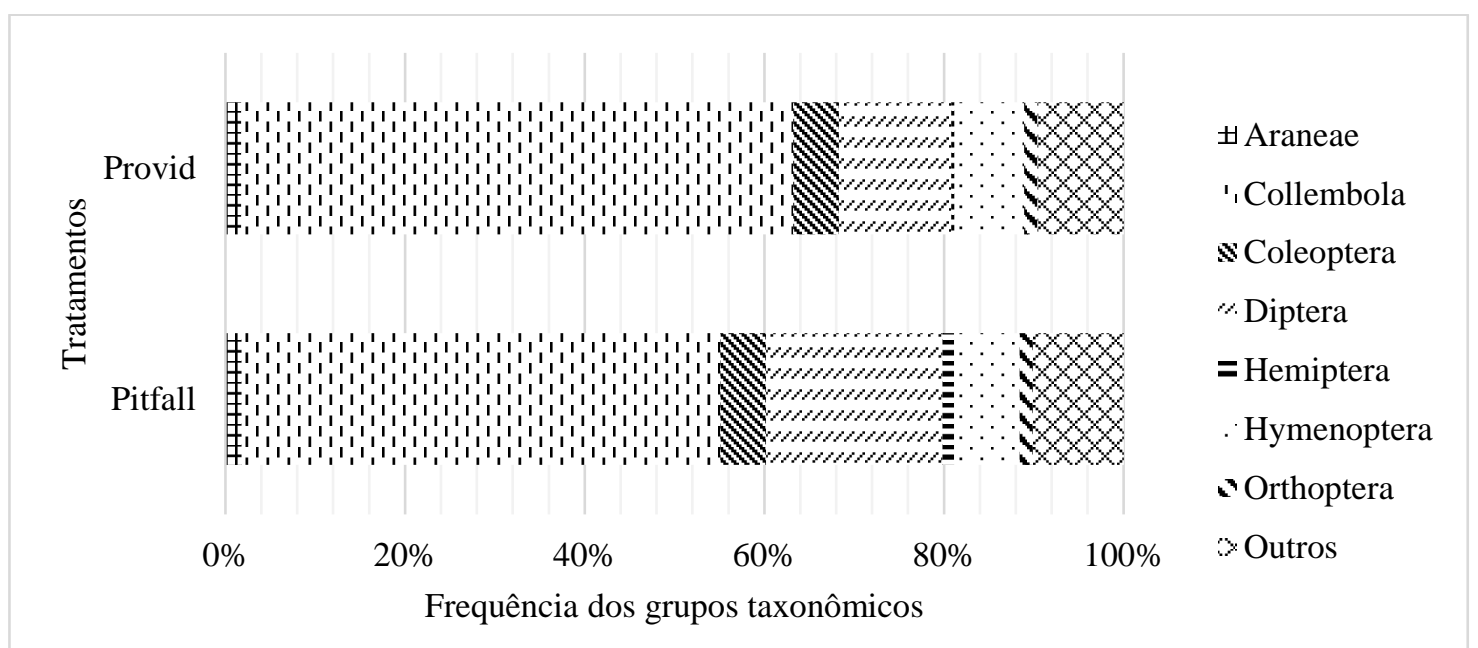

Figura 3. Frequência dos grupos taxonômicos da fauna epígea, amostrados pelas metodologias de Pitfall e Provid, instaladas na floresta ciliar do Câmpus da UTFPR, em Dois Vizinhos - PR.

Figure 3. Frequency of the taxonomic groups of the epigeal fauna, sampled by the methodologies of Pitfall and Provid, installed in the ciliary forest of the Campus of the UTFPR, in Dois Vizinhos - PR.

Em relação à Análise de Componentes principais (ACP), o componente principal 1 (PC1) explicou 93,23\% da variância dos dados e o componente principal 2, explicou 4,77\%, resultando em uma variância acumulada de 98\%. Não houve separação dos tratamentos avaliados, no entanto, destaca-se os vetores indicando os grupos taxonômicos mais abundantes, sendo Collembola e Diptera (Figura 4). 


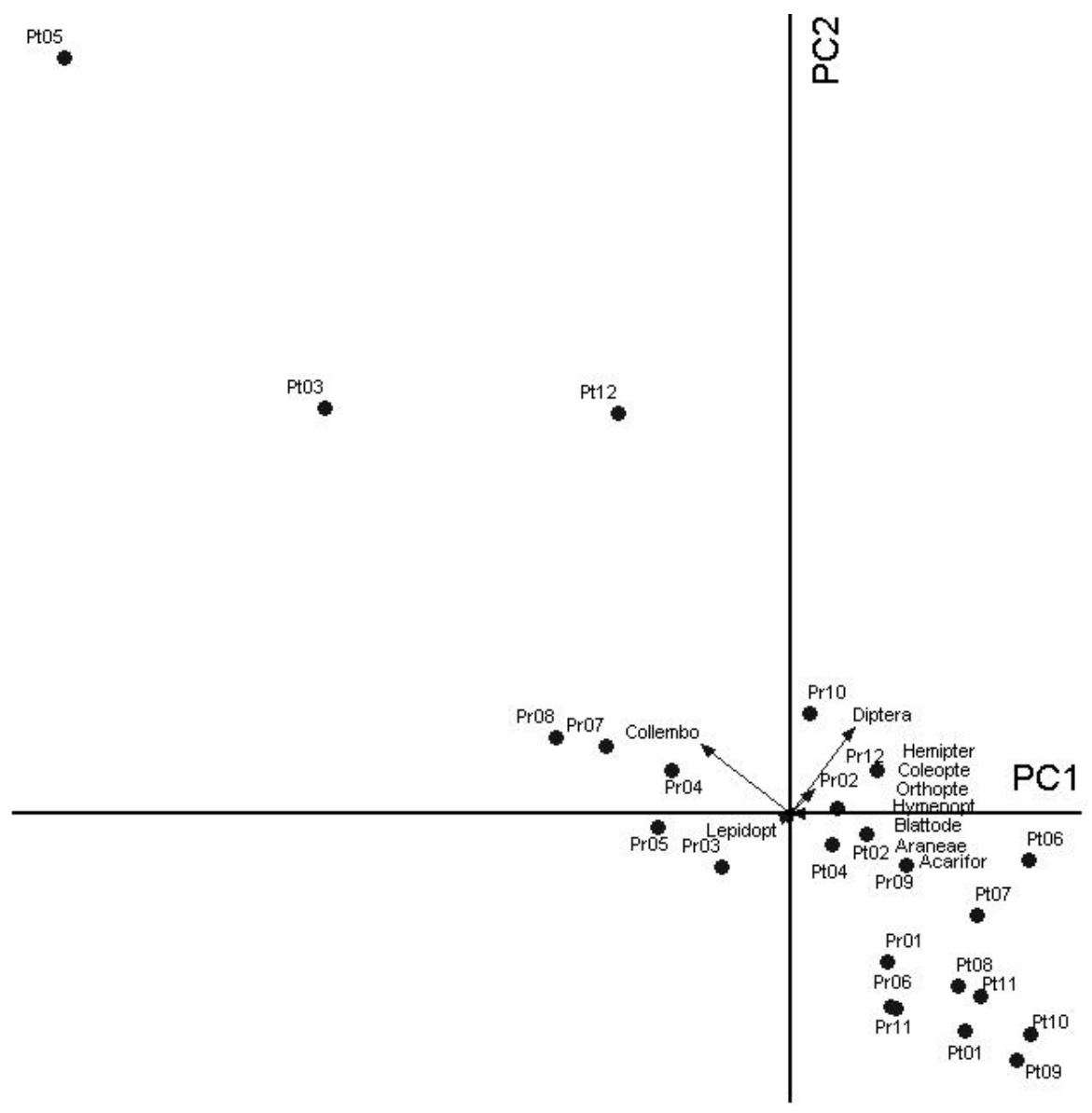

Figura 4. Análise de Componentes principais (ACP) para os diferentes grupos taxonômicos da fauna epígea nas diferentes armadilhas avaliadas no Sudoeste do Paraná. Pt: Pitfall; Pr: Provid.

Figure 4. Analysis of Components main for the different taxonomic groups of the epigeal fauna in the evaluations in the Southwest of Paraná. Pt: Pitfall; Pr: Provid

\section{DISCUSSÃO}

Considerando a época de coleta realizada no outono, caracterizada por temperaturas mais amenas, é possível que este fator abiótico tenha influenciado na comunidade epígea, diminuindo sua atividade biológica e consequentemente a riqueza e abundância de organismos amostrados. Diferenças na abundância de indivíduos da fauna epigéica foram reportadas por Bartz et al. (2014) entre as estações de inverno (menor abundância) e verão (maior abundância). Em campo nativo no sudoeste do estado do Rio Grande do Sul, em coleta da fauna realizada no 
mês de fevereiro (verão), a abundância relativa dos grupos foi maior comparada àquela observada em coleta realizada em maio (outono) (ROVEDDER et al., 2009).

Os melhores valores dos índices ecológicos foram obtidos com a armadilha Pitfall, pois verifica-se melhor distribuição dos indivíduos na comunidade amostrada apesar desta também apresentar dominância de alguns grupos taxonômicos, como Collembola, Hymenoptera e Diptera. Ainda, como observado no presente estudo, o aumento na frequência de alguns grupos pode resultar em menor equitabilidade, levando a menor diversidade (CUNHA NETO et al., 2012), conforme observado na armadilha Provid, para qual obteve-se maior porcentagem destes grupos dominantes $(89,3 \%)$ em relação a Pitfall $(88,1 \%)$. O aumento da densidade e a redução da riqueza da fauna contribuem para a redução da equitabilidade e, consequentemente, do índice de Shannon. Por outro lado, a diminuição da densidade e aumento da riqueza favorecem o incremento da equitabilidade entre grupos (MOÇO et al., 2005).

Ludwig et al. (2012) observaram que, em área com elevada ocorrência de colêmbolos, o índice de Shannon foi menor que em áreas onde tal predominância não ocorreu. Diversos autores também verificaram a abundância de colêmbolos e himenópteros em solos de florestas tropicais (MOÇO et al., 2010; OLIVEIRA FILHO et al., 2014; OLIVEIRA FILHO et al., 2015). Além disso, colêmbolos, juntamente com os ácaros, são os artrópodes edáficos mais abundantes, podendo haver no solo, milhares de indivíduos por metro quadrado (PALACIOS-VARGAS et al., 2007; RIEFF et al., 2014). Os colêmbolos estão entre os invertebrados mais abundantes no sistema solo-serapilheira, sendo decompositores primários e secundários, responsáveis pela fragmentação da matéria orgânica em partes menores, processo que favorece outros decompositores tais como fungos e bactérias (MOREIRA; SIQUEIRA, 2006).

A ordem Diptera foi a segunda mais abundante, apesar não serem considerados insetos edáficos em sua fase adulta, são abundantes em áreas florestais e acabam caindo nas armadilhas. Em um estudo na mata atlântica do Rio Grande do Sul, utilizando armadilhas Pitfall, Copatti e Daudt (2009) também amostraram uma grande abundância de Diptera, sendo esse o grupo taxonômico 
mais frequentemente amostrado, devido a atração pela solução utilizada nas armadilhas.

Em relação a ordem Hymenoptera, todos os indivíduos amostrados pertenciam a família Formicidae. Copatti e Daudt (2009) também encontraram apenas formigas como representantes dessa ordem, sendo um dos grupos mais abundantes coletados. As formigas, além de dominantes em ecossistemas terrestres, tanto em abundância e biomassa como em funções ecológicas exercidas, também são importantes engenheiras do solo. Dentre as principais funções desempenhadas, estão: a movimentação de solo para a superfície, aeração do solo, revolvimento e infiltração de água, ciclagem de nutrientes, dispersão de sementes, herbívoria, polinização e atividades de engenharia em seus ninhos (KORASAKI et al., 2013).

Em relação ao índice de Margalef, o qual expressa à riqueza de espécies verifica-se elevada similaridade entre o método Pitfall $(1,24)$ o método Provid $(1,27)$. Embora verifique-se esta sutil diferença Richter et al., (2012), destaca que valores menores que 2,0 indicam baixa riqueza, demonstrando que ambas as áreas se encaixam na mesma classificação.

Considerando a análise de componentes prinicipais, observa-se que não há separação dos grupos em relação ao método, indicando que dentre os organismos passíveis de coleta pelos métodos testados, nenhum deles favorece a captura de um grupo em detrimento de outro.

\section{CONCLUSÕES}

Não houve diferenças significativas na identificação da comunidade epígea em fragmento florestal por meio de diferentes métodos de coleta

O método de captura Pitfall pode ser usado com a mesma eficiência que o método Provid, sendo também mais prático e fácil de manejar, já que é um recipiente fechado, que pode ser levado a campo já contendo a solução conservante facilitando a retirada e envio das amostras do campo ao laboratório. 


\section{REFERÊNCIAS BIBLIOGRÁFICAS}

ALVARES, C. A.; STAPE, J. L.; SENTELHAS, P. C.; DE MORAES GONÇALVES, J. L.; SPAROVEK, G. Köppen's climate classification map for Brazil. Meteorologische Zeitschrift, Stuttgart, v. 22, n. 6, p. 711-728, 2013.

ANTONIOLLI, Z. I.; CONCEIÇÃO, P. C.; BÖCK, V.; PORT, O.; SILVA, D. M.; SILVA, R. F. Método alternativo para estudar a fauna do solo. Ciência Florestal, Santa Maria, v. 16, n. 4, p. 407-417, 2006.

ARAÚJO, C. C.; NOMELINI, Q. S. S.; PEREIRA, J. M.; LIPORACCI, S. N. H.; KATAGUIRI, V. S. Comparação da abundância de invertebrados de solo por meio da estimação intervalar encontrados em diferentes ambientes na cidade de Ituiutaba MG. Bioscience Journal, Uberlândia, v. 26, n. 5, p. 817-823, 2010.

BARDGETT, R. D.; VAN DER PUTTEN, W. H. Belowground biodiversity and ecosystem functioning. Nature, v. 515, p. 505-511, Nov. 2014.

BARETTA, D.; BROWN, G. G.; CARDOSO, E. J. B. N. Potencial da macrofauna e outras variáveis edáficas como indicadores de qualidade do solo em áreas com Araucaria angustifolia. Acta Zoológica Mexicana, Veracruz, v. 2 p. 135-150, 2010.

BARETTA, D.; SANTOS, J. C. P.; SEGAT, J. C.; GEREMIA, E. V.; OLIVEIRA FILHO, L. C. I. de.; ALVES M. V. Fauna edáfica e qualidade do solo. In: KLAUBERG FILHO, O.; MAFRA, A.L. (Org.). In: Tópicos em Ciências do Solo. Viçosa: Sociedade Brasileira de Ciência do Solo, v. 7, p. 119-170, 2011.

BARTZ, M. L. C.; BROWN, G. G.; ORSO, R.; MAFRA, A. L.; BARETTA, D. The influence of land use systems on soil and surface litter fauna in the western region of Santa Catarina. Revista Ciência Agronômica, Fortaleza, n. 45, p. 880-887, 2014.

BHERING, L. L. Rbio: A Tool For Biometric And Statistical Analysis Using The R Platform. Crop Breeding and Applied Biotechnology, v. 17, p. 187-190, 2017.

COPATTI, C. E.; DAUDT, C. R. Diversidade de artrópodes na serapilheira em fragmentos de mata nativa e Pinus elliottii (Engelm. Var elliottii). Ciência e Natura, 
Santa Maria, v. 31, n. 1, p. 95-113, 2009.

CORREIA, M. E. F.; ANDRADE, A. G. Formação de serapilheira e ciclagem de nutrientes. In: SANTOS, G. A.; SILVA, L.; CANELLAS, L. P. \& CAMARGO, F. A. O. Fundamentos da matéria orgânica do solo: Ecossistemas tropicais e subtropicais. 2.ed. Porto Alegre, Metrópole, 2008. p.137-158.

CUNHA NetO, F. V.; CORREIA, M. E. F.; PEREIRA, G. H. A.; PEREIRA, M. G.; LELES, P. S. S. Soil fauna as an indicator of soil quality in forest stands, pasture and secondary forest. Revista Brasileira de Ciência do Solo, Viçosa, n. 36, p. 14071417, 2012.

EMPRESA BRASILEIRA DE PESQUISA AGROPECUÁRIA. Sistema Brasileiro de Classificação de Solos, 3. ed. EMBRAPA-SPI, Brasília, 2013.

FREIRE, L. R.; ARAUJO, E. S.; BERBARA, R. L. L. Tempo de Captura de Organismos da Mesofauna do Solo e seus Reflexos na Interpretação de Índices da Comunidade Edáfica. Revista Brasileira de Ciência do Solo, Viçosa, v. 39, n. 5, 2015.

HAMMER, O. Paleontological Statistics. Version 2.16. Manual de Referência. Natural History Museum. University of Oslo. 1999-2012. 187p.

HUERTA, E.; WAL, H. Soil macroinvertebrates abundance and diversity in home gardens in Tabasco, Mexico, vary with soil texture, organic matter and vegetation cover. European Journal of Soil Biology. v. 50, p. 68-75, 2012.

KORASAKI, V.; MORAIS, J. W.; BRAGA, R. F.; Macrofauna. In: MOREIRA, F. M. S.; CARES, J. E.; ZANETTI, R..; STURMER, S. L. O ecossistema solo: componentes, relações ecológicas e feitos na produção vegetal. Lavras: Ed. UFLA, 2013. 352 p.

LUDWIG, R. L.; PIZZANI, R.; SCHAEFER, P. E.; GOULART, R. Z.; LOVATO, T. Efeito de diferentes sistemas de uso do solo na diversidade da fauna edáfica na região central do Rio Grande do Sul. Enciclopédia Biosfera, Jandaia, n. 8, p. 485495, 2012. 
MAGURRAN, A. E. Medindo a diversidade biológica. Curitiba: Editora da UFPR; 2011.

MCCUNE, B; MEFFORD, M. J. Multivariate Analysis of Ecological Data. Version 4.25. MjM Software, Gleneden Beach, Oregon, U.S.A, 1999.

MOÇO, M. K. S.; GAMA-RODRIGUES, E. F.; GAMA-RODRIGUES, A. C.; CORREIA, M. E. F. Caracterização da fauna edáfica em diferentes coberturas vegetais na Região Norte Fluminense. Revista Brasileira de Ciência do Solo, Viçosa, n. 29, p. 555-564, 2005.

MOÇO, M. K. S.; GAMA-RODRIGUES, E. F.; GAMA-RODRIGUES, A. C.; MACHADO, R. C. R.; BALIGAR, V. C. Relationships between invertebrate communities, litter quality and soil attributes under different cacao agroforestry systems in the south of Bahia, Brazil. Applied Soil Ecology, v. 46, n. 3, p. 347-354, 2010.

MOREIRA, F. M. S.; SIQUEIRA, J. O. Microbiologia e bioquímica do solo. Lavras: UFLA, 2006. 729p.

OLIVEIRA FILHO, L. C. I.; BARETTA, D.; SANTOS, J. C. P. Influência dos processos de recuperação do solo após mineração de carvão sobre a mesofauna edáfica em Lauro Müller, Santa Catarina, Brasil. Biotemas, Florianópolis, 27, n. 2, p. 69-77, 2014.

OLIVEIRA FILHO, L. C. I.; BARETTA, D.; VIAPIANA, C. M.; SANTOS, J. C. P. Mesofauna de solo construído em área de mineração de carvão. Revista de Ciências Agroveterinárias, Lages, v. 14, n. 1, p. 55-64, 2015.

PALACIOS-VARGAS, J. G.; CASTAÑO-MENESES, G.; GÓMEZ-ANAYA, J. A.; MARTÍNEZ-YRIZAR, A.; MEJÍA-RECAMIER, B. E.; MARTÍNEZ-SÁNCHEZ, J. Litter and soil arthropods diversity and density in a tropical dry forest ecosystem in Western Mexico. Biodiversity and Conservation, v. 16, n. 13, p. 3703-3717, 2007.

PIELOU, E. C. The measurement of diversity in different types of biological 
collections. Journal of Theoretical Biology, v. 13, p. 131-144, 1966.

RICHTER, C.; PEITER, M. X.; ROBAINA, A. D.; SOUZA, A. R. C.; FERRAZ, R. C.; DAVID, A. F. Levantamento da arborização urbana de Mata/RS. Revista da Sociedade Brasileira de Arborização Urbana, v.7, n.3, p.84-92, 2012.

RIEFF, G. G.; NATAL-DA-LUZ, T.; SOUSA, J. P.; LUIZ, E.; SÁ, S. De. Diversity of Springtails and Mites of a Native Forest in Southern Brazil: Relationship with the Indices of Temperature and Precipitation in the Native Environment. International Journal of Emerging Technology and Advanced Engineering, Madhya Pradesh, v. 4, n. 9, p. 684-692, 2014.

ROVEDDER, A. P. M., ELTZ, F. L. F., DRESCHER, M. S., SCHENATO, R. B.; ANTONIOLLI, Z. I. Organismos edáficos como bioindicadores da recuperação de solos degradados por arenização no Bioma Pampa. Ciência Rural, Santa Maria, v. 39, n.4, p. 1061-1068, 2009.

SHANNON, C. E. A mathematical theory of communication. The Bell System Technical Journal, v. 27, p. 379-423, 1948.

SIMPSON, E. H. Measurement of diversity. Nature, v. 163, 1949. 688 p.

WALL, D. H.; BARDGETT, R. D. V.; HERRICK, J. E.; JONES, H.; SIX, J.; RITZ, K.; STRONG, D. R.; VAN DER PUTTEN, W. H. Soil Ecology and Ecosystem Services. Oxford Univ. Press, 2012. 